IN THE NEWS

\title{
The Lasker goes to Michael Hall
}

The 2017 Albert Lasker Basic Medical Research Award goes to Michael N. Hall (Biozentrum, University of Basel, Switzerland) "for discoveries concerning the nutrient-activated TOR proteins and their central role in the metabolic control of cell growth" (Lasker Foundation, 6 Sep 2017).

TOR (target of rapamycin) proteins are evolutionarily conserved Ser/Thr protein kinases that sense the cellular energy status and the availability of nutrients and other growth signals and integrate these signals to regulate metabolic pathways that drive cell growth.

Hall discovered the TOR1 and TOR2 proteins in budding yeast in the 1990s. His discoveries revealed that cell growth is an actively regulated process, which came as a surprise, as previously cell growth was thought to be driven by spontaneous increase in cell size (owing to increased protein and lipid synthesis), when nutrients are available. Initially, Hall and colleagues were interested in how the immunosuppressant drug rapamycin functions, and found that it blocked cell proliferation in yeast cells. This enabled them to isolate TOR 1 and TOR2, the deletion of which mimicked the effects of rapamycin, inhibiting both cell division and cell growth.

The mammalian counterpart, mTOR, was identified by several research groups for its ability to bind to rapamycin, and subsequent work over the years from the Hall laboratory and others has revealed the importance of the mTOR signalling network in animal physiology, development and ageing, as well as its crucial role in diseases such as diabetes and cancer.
Thus, Hall opened the doors to a big research field, unravelling fundamental mechanisms of growth and development. Although many of the intricate mechanisms underlying mTOR function have been identified, many questions remain, including how mTOR interacts with upstream regulators and other signalling pathways, how it affects many downstream processes, and how mTOR deregulation can lead to disease.

Kim Baumann

FURTHER READING Heitman, J. et al. Targets for cell cycle arrest by the immunosuppressant rapamycin in yeast. Science 253, 905-909

(1991) | Kunz, J. et al. Target of rapamycin in yeast, TOR2, is an essential phosphatidylinositol kinase homolog required for $\mathrm{G} 1$ progression. Cell 73 , 585-596 (1993) | Barbet, N. C. et al. TOR controls translation initiation and early $\mathrm{G} 1$ progression in yeast. Mol. Biol. Cell 7, 25-42 (1996) |Beck, T. \& Hall, M. N. The TOR signalling pathway controls nuclear localization of nutrient-regulated transcription factors. Nature 402, 689-692 (1999) | Loewith, R. et al. Two TOR complexes, only one of which is rapamycin sensitive, have distinct roles in cell growth control. Mol. Cell 10, 457-468 (2002) |Shimobayashi, M. \& Hall, M. N. Making new contacts: the mTOR network in metabolism and signalling crosstalk. Nat. Rev. Mol. Cell Biol. 15, 155-162 (2014) 